\title{
Observations of isolated enterocytes and of their subcellular components using transmission and scanning electron microscopy
}

\author{
M. N. MARSH, ${ }^{1}$ T. J. PETERS, ${ }^{2}$ AND A. C. BROWN \\ From the MRC Intestinal Malabsorption Group, Department of Medicine, Royal Postgraduate Medical School, \\ London, and Unilever Research Laboratory, Isleworth, Middlesex
}

SUMMARY Isolated epithelial cells and their subcellular organelles were examined both by transmission and by scanning electron microscopy. It was shown that scanning electron microscopy is particularly useful for displaying the three-dimensional structure of the brush border. Furthermore, it has been possible to observe the basal aspect of separated epithelium for the first time. Studies on the scanning electron microscopic appearance of isolated nuclei, mitochondria, and lysosomes did not reveal any evidence of surface structure.

The use of the scanning electron microscope for studying intestinal epithelium has already been described in this journal (Marsh and Swift, 1969). Several techniques are now available which may be used to prepare isolated intestinal epithelial cells (enterocytes), and subcellular fractions of these cells may also be obtained in relatively pure form. In this paper the appearances of some of these fractions have been studied using scanning electron microscopy and compared with those observed through the transmission electron microscope. The results of these investigations are described in relation to existing knowledge of the structure of the intestinal epithelium.

\section{Materials and Methods}

Isolated epithelial cells were prepared from the jejunum of adult rats and guinea pigs. Pieces of small intestinal mucosa were incubated either in $\mathrm{Ca}^{2+}$ - and $\mathrm{Mg}^{2+}$-free Tyrode's solution containing $0 \cdot 1 \mu \mathrm{g} / 100$ $\mathrm{ml}$ phospholipase $\mathrm{C}$ or in buffered $10 \mathrm{mM}$ EDTA (Evans, Wrigglesworth, Burdett, and Pover, personal communication). The cells were harvested by gentle centrifugation, and were washed and resuspended in buffered $0 \cdot 2 \mathrm{M}$ mannitol.

Brush borders were prepared by the methods of Eichholz and Crane (1965) and of Hübscher, West,

Received for publication 15 April 1971.

'Present address: Department of Medicine, Boston University Medical Center, 750 Harrison Avenue, Boston, Mass. 02118, USA. 'Present address: Department of Biochemical Cytology, The Rockefeller University, New York, New York, 10071, USA. and Brindley (1965). A combined mitochondrial and lysosomal fraction was prepared by the technique of Hübscher et al (1965), and isolated nuclei by the method of Haussler, Thompson, and Norman (1969).

For scanning electron microscopy drops of each suspension were added to $5 \%$ glutaraldehyde in $0.1 \mathrm{M}$ phosphate buffer (Sabatini, Bensch, and Barrnett, 1963) or buffered $\mathrm{OsO}_{4}$ in saturated $\mathrm{HgCl}_{2}$ solution (Parducz, 1967), fixed for one hour, and lyophilized (Small and Marszalik, 1969). The dried material was vacuum-coated with carbon and silver and examined in a Cambridge mark II scanning electron microscope.

For transmission electron microscopy, drops of each suspension were added to buffered $5 \%$ glutaraldehyde (Sabatini et al, 1963) and fixed for one hour, dehydrated in a graded ethanol series, and embedded in Araldite resin. Thin sections were stained with uranyl magnesium acetate and lead citrate (Frasca and Parks, 1965) and examined in a AEI-EM6 transmission electron microscope.

\section{Results}

ISOLATED ENTEROCYTES

Isolated epithelial cells, prepared by both techniques, were wider at their apical margins and tapered towards their basal poles. Those obtained after incubation for $30 \mathrm{~min}$ with phospholipase C (Fig. 1a) had a relatively smooth surface which was pitted with numerous small indentations. The brush border was well preserved, and, although the microvilli had 


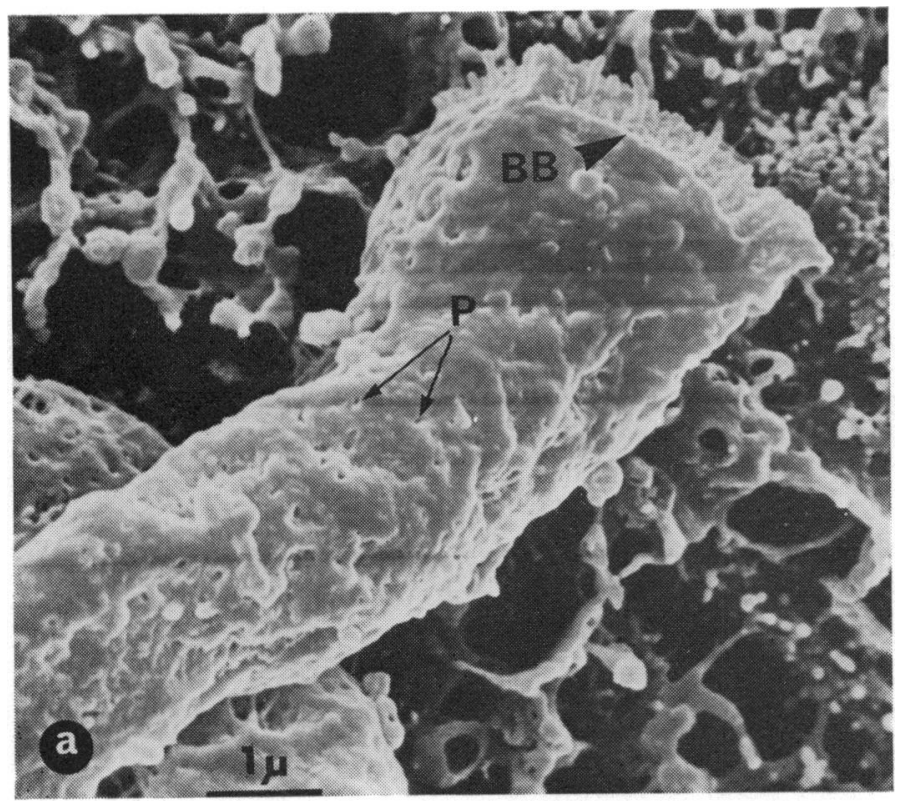

Fig. 1 Scanning electron microscopic appearance of cells prepared by the phospholipase $C$ method: incubation time 30 minutes.

a A single enterocyte showing well preserved brush border BB, and smooth lateral surface indented by numerous pits $P$. $b$ Detail of brush border, showing panoramic view of microvilli. Beading of microvilli (arrows) at edge of cell, is clearly visible: note the 'drum-stick' appearance of some of these microvilli (large arrow heads) which could be due to blobs of extraneous coat material.

Fig. 1a.

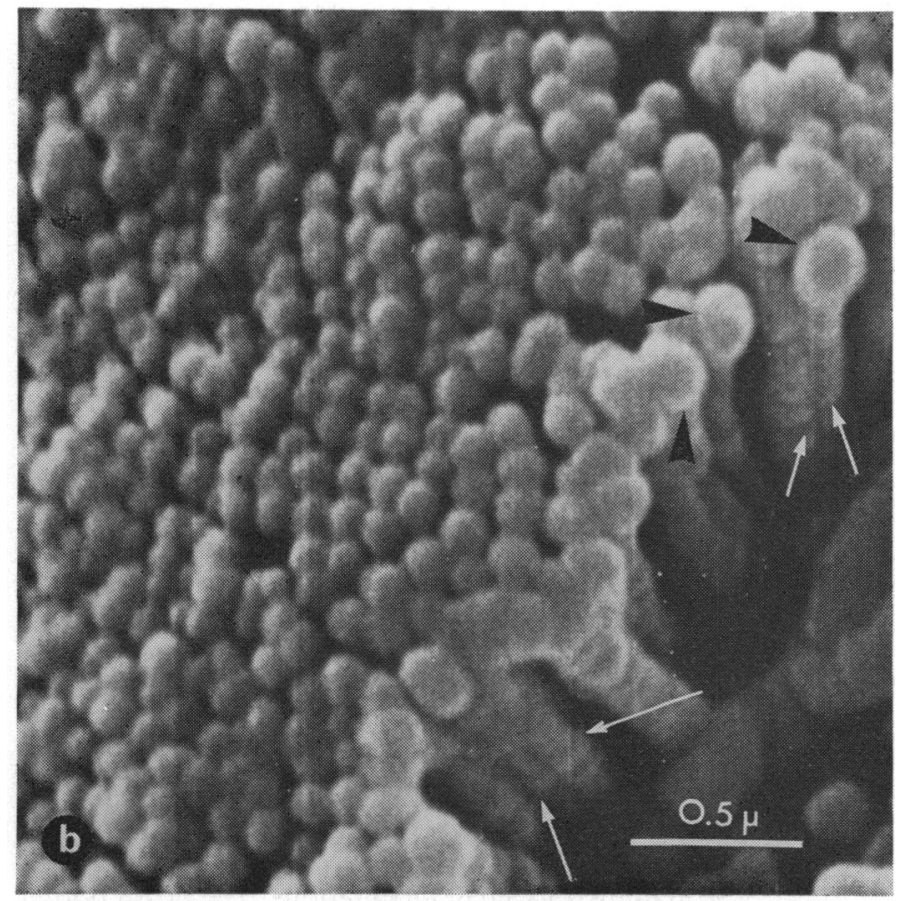

Fig. 1b. 


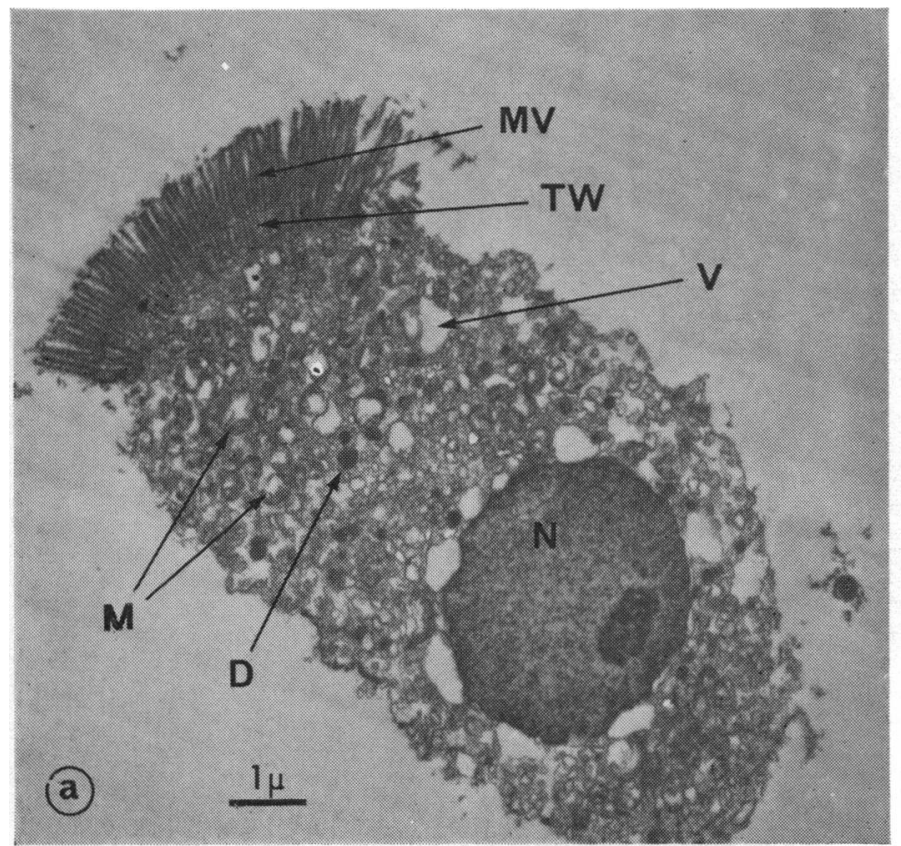

Fig. 2 Enterocytes prepared by EDTA method; 12 min incubation.

a Transmission electron microscope appearance showing intact brush border, $M V$, and terminal web, $T W$. Remaining cytoplasm contains numerous vacuoles, $V$, disorganized mitochondria, $M$, and dense bodies, $D$, which are probably lysosomes. The membrane of nucleus, $N$, is crenated and partially separated from cytoplasm. The lateral cell margin is irregular, and in parts lacks a recognizable membrane profile. b Scanning electron microscope appearance shows a cell with markedly irregular surface texture, but intact brush border

Fig. 2a.

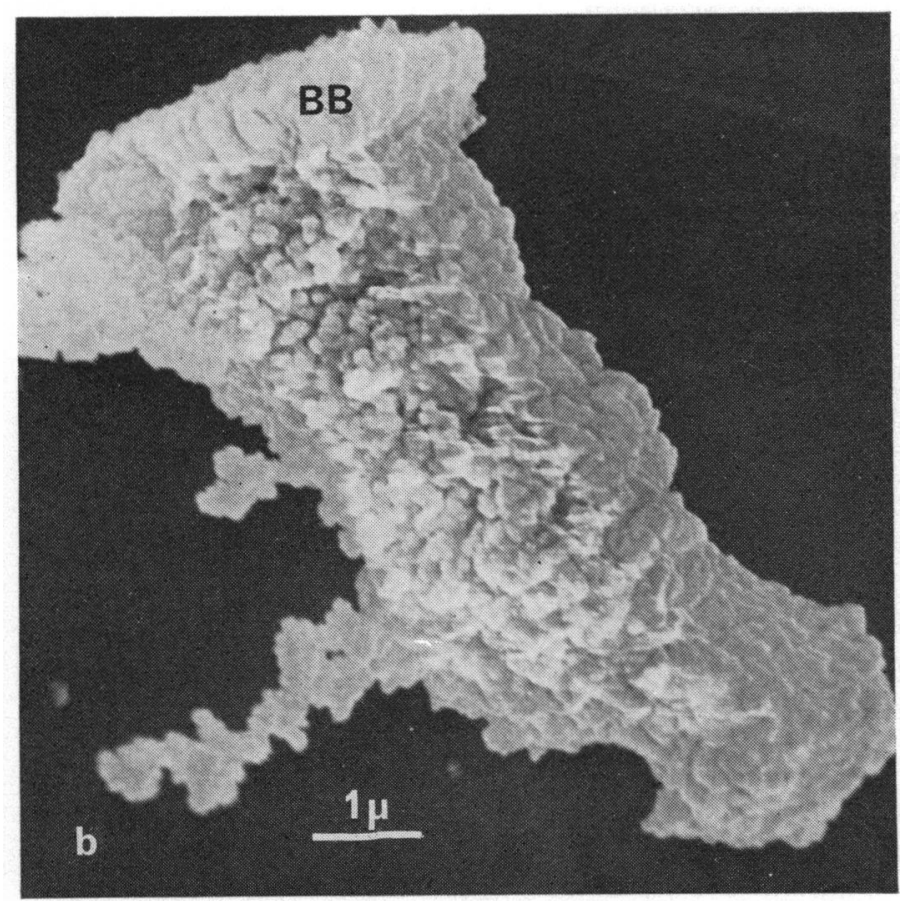

Fig. 2b. 


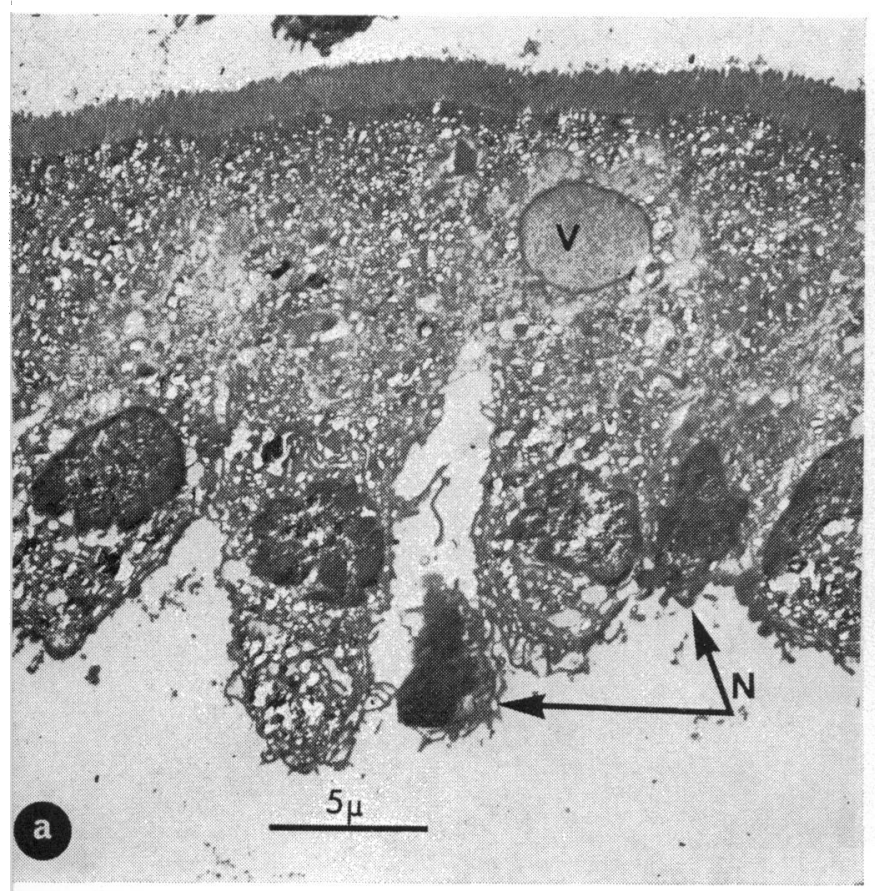

Fig. 3a.

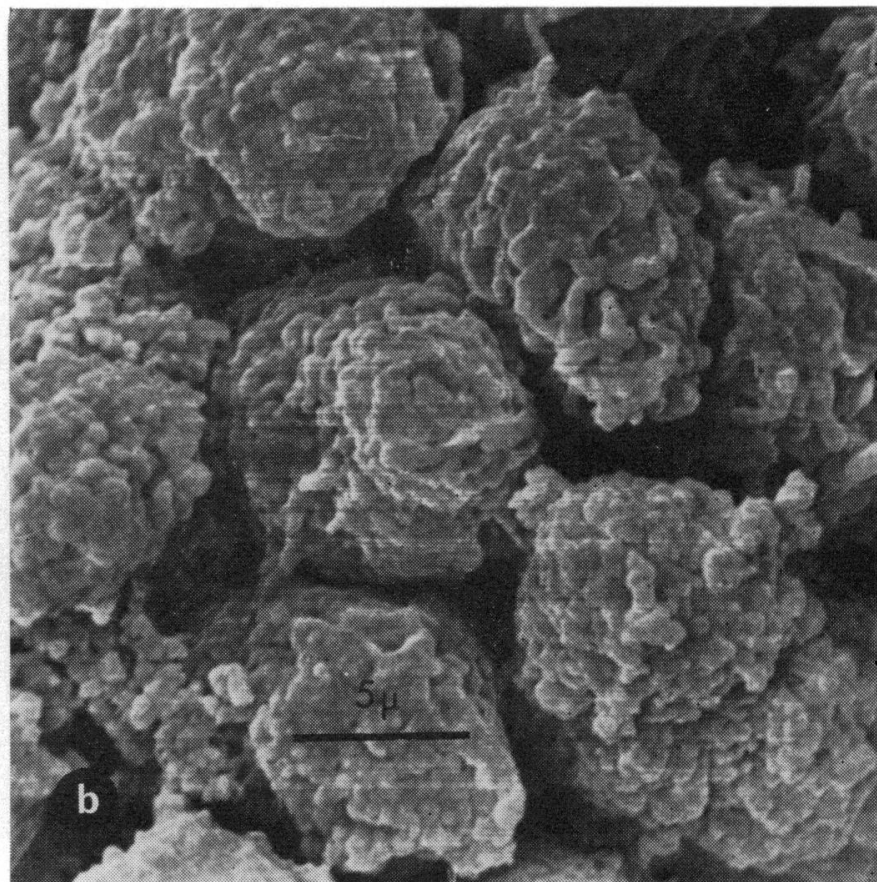

Fig. 3b.

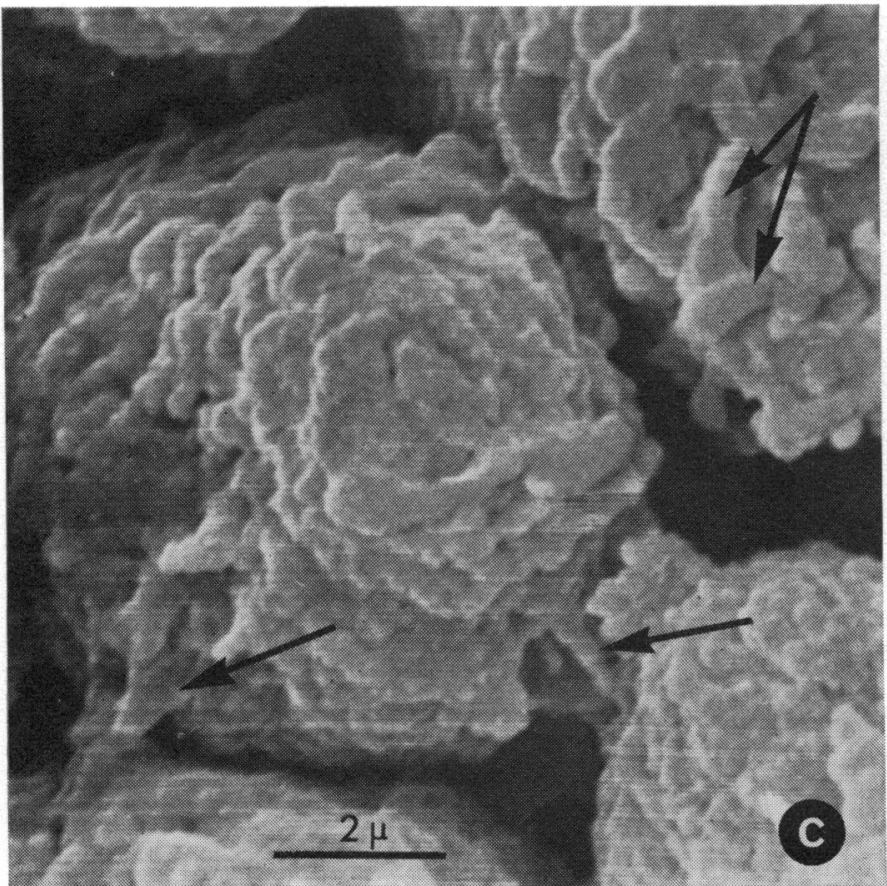

Fig. 3 EDTA technique.

a Section of plaque of detached enterocytes, which are similar in appearance to that illustrated in Figure $2 a$. In addition note large vacuole, $V$, and displaced nuclei, $N$. $b$ Undersurface of plaque as seen in scanning electron microscope. Although each cell has an irregular contour, numerous intercellular projections are visible, as shown in detail in $c$.

Fig. 3c. 


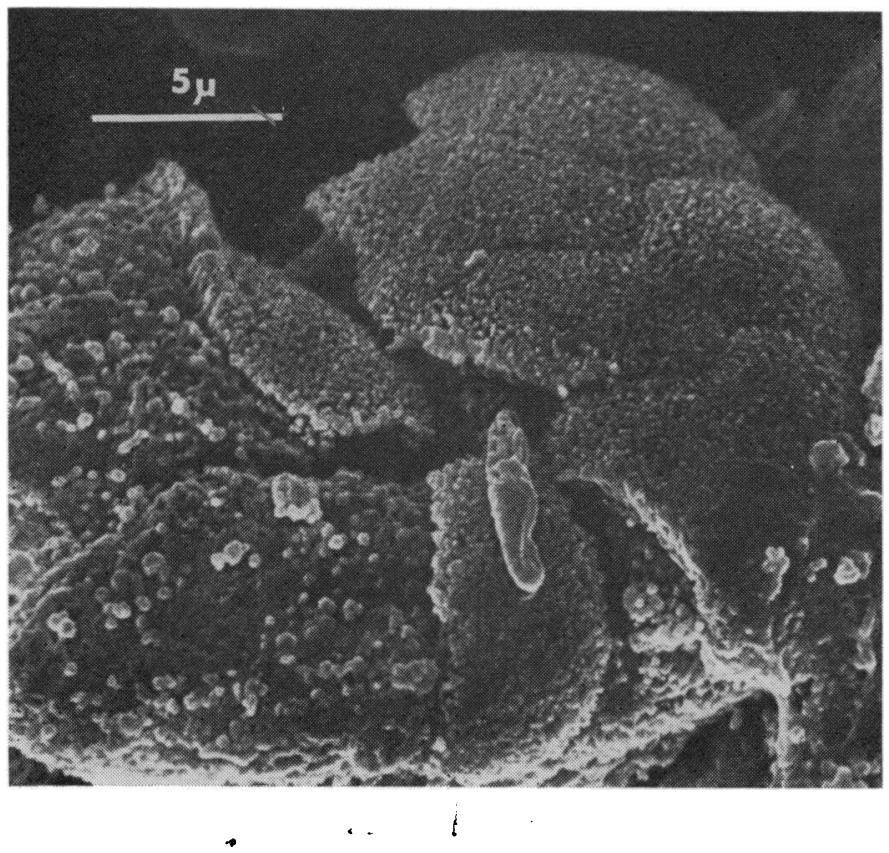

Fig. 4 Phospholipase $C$ method, 30 min incubation, scanning electron microscope. A group of six cells lies free on the exposed basement lamina. Their hexagonal outline is clearly visible and the microvilli are easily distinguished. In the foreground, two other enterocytes have been partially separated, but the brush border is preserved as an intact cap at the apical pole.

a beaded appearance (Fig. 1b), their regular packing was dramatically displayed. The brush border of cells obtained by the EDTA method showed less beading of individual microvilli (Fig. 2a). The nuclei of the latter cells were frequently contracted and their membranes were crenated: in several cells, the nuclei were either absent or appeared just beneath the brush border. The lateral surface of cells prepared by the EDTA method was rougher than that of cells obtained by the phospholipase $\mathrm{C}$ method (Fig. 2b) due to the numerous vesicular protrusions of their cytoplasm.

Several clumps of epithelial cells were also obtained by each method. The appearance of these fragments of epithelium in section (Fig. 3a) showed that the basal portions were separated from each other. Using the scanning electron microscope, it was possible to observe the epithelium from its undersurface (Fig. 3b). The basal pole of each cell was rounded, and although it was not possible to recognize desmosomal attachments, large intercellular connexions were frequently seen (Fig. 3c). When viewed from above (Fig. 4), the hexagonal outline of the enterocytes was clearly visible, and furthermore the plate-like structure of the brush border was strikingly displayed. In the group illustrated two cells are shown partially detached from their neighbours, but it is not possible to identify the terminal web or the adjacent tight junctions.

\section{SUBCELLULAR FRACTIONS}

\section{Brush borders}

Brush borders, prepared by the technique of Eichholz and Crane (1965), were relatively clean and consisted of a strip of microvilli attached to the terminal web (Fig. 5a). Contamination with other subcellular organelles was minimal. In the scanning electron microscope the tips of the microvilli could be distinguished easily (Fig. 5b). Brush borders prepared by the technique of Hubscher et al (1965) showed marked beading of the microvilli and were contaminated by other subcellular organelles, particularly lysosomes (Figs. 6a and 6b).

\section{Mitochondria and lysosomes}

Mitochondria had the typical double membranes and cristae whereas the lysosomes were more variable in shape and were enclosed by a single membrane (Fig. 7a). In the scanning electron microscope these organelles appeared as spherical objects of varying diameter (Fig. 7b). It was not possible to distinguish between them and no surface features could be identified at this level of resolution.

\section{Nuclei}

Well preserved nuclei were obtained with little contamination by other subcellular organelles or debris (Fig. 8a). No evidence of nuclear pores could 


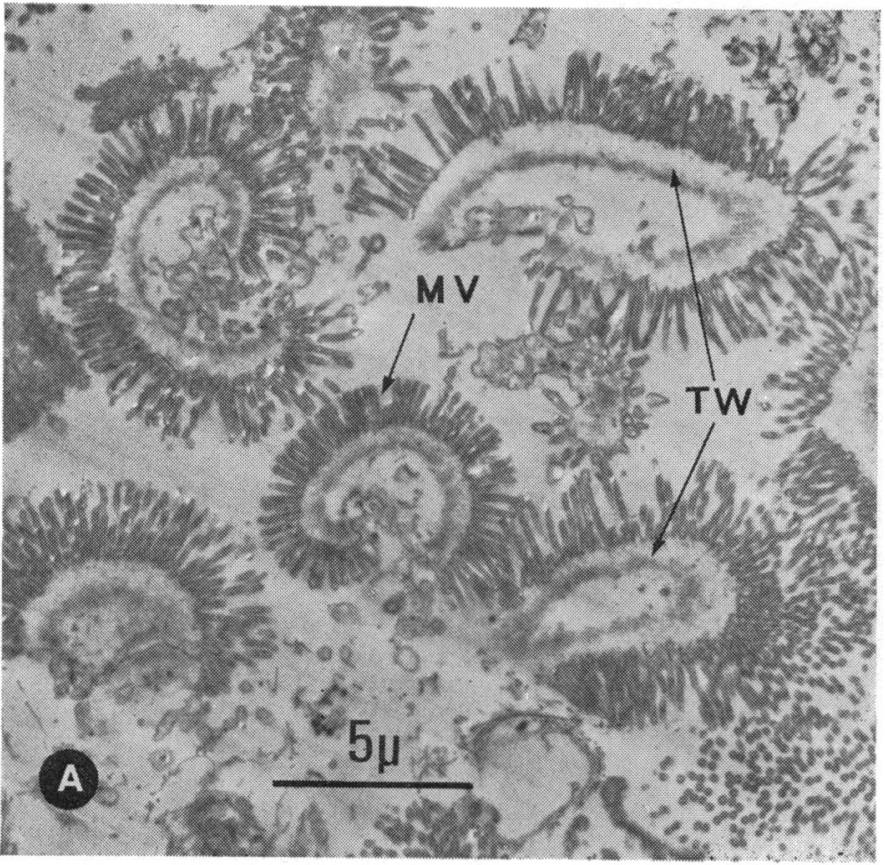

Fig. 5a.
Fig. 5 Brush border preparations (Eichholz and Crane, 1965).

a well preserved microvilli, $M V$, attached to terminal web, $T W$, seen in the transmission electron microscope.

$b$ Discrete tips of microvilli observed through the scanning electron microscope.

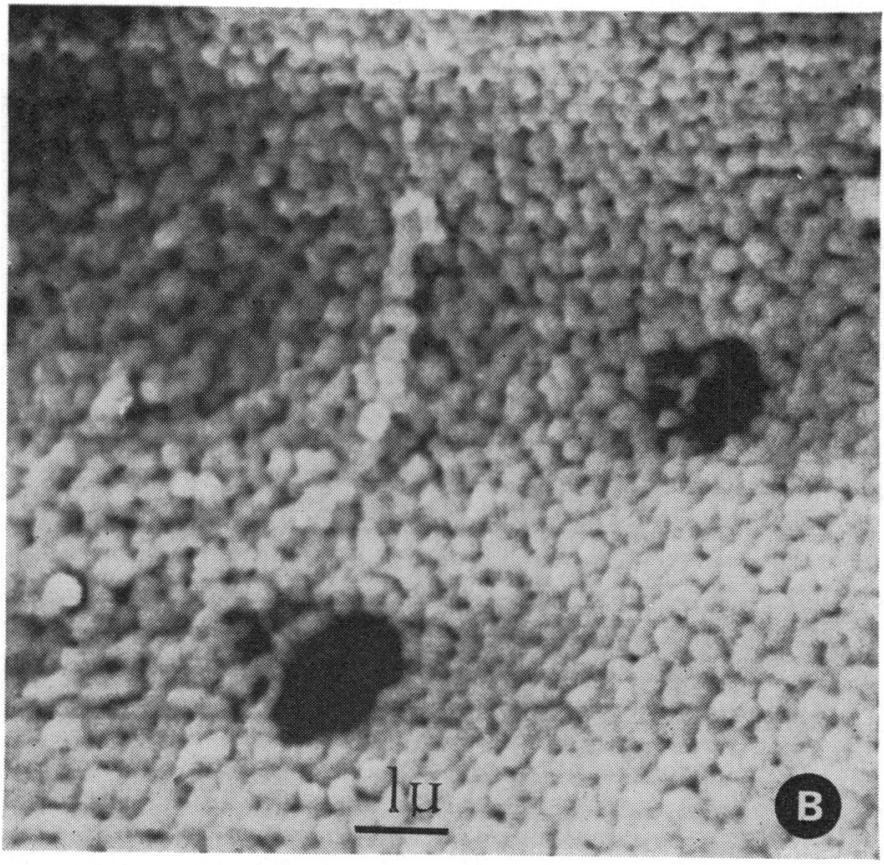

Fig. 5b. 


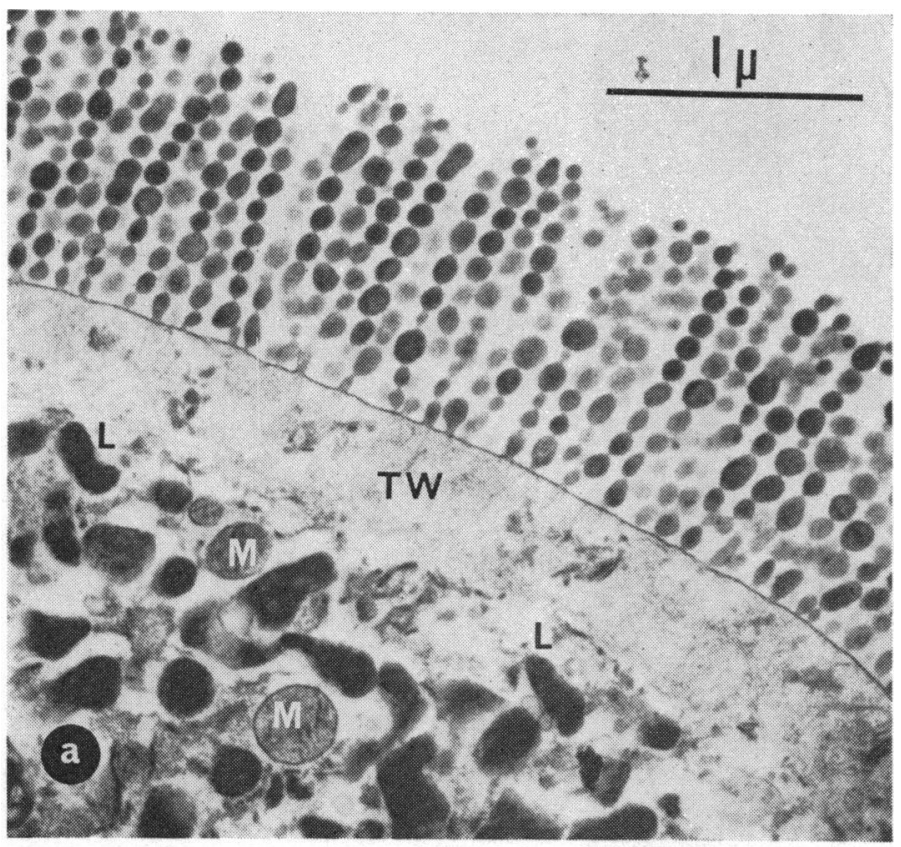

Fig. 6 Brush border preparations (Hübscher et al, 1965).

a In transmission electron microscope marked beading of microvilli is shown, and beneath the terminal web, TW, contaminating cell components including lysosomes, $L$, and mitochondria, $M$, are present.

$b$ Discrete microvilli demonstrated in similar preparations using the scanning electron microscope.

Fig. 6a.

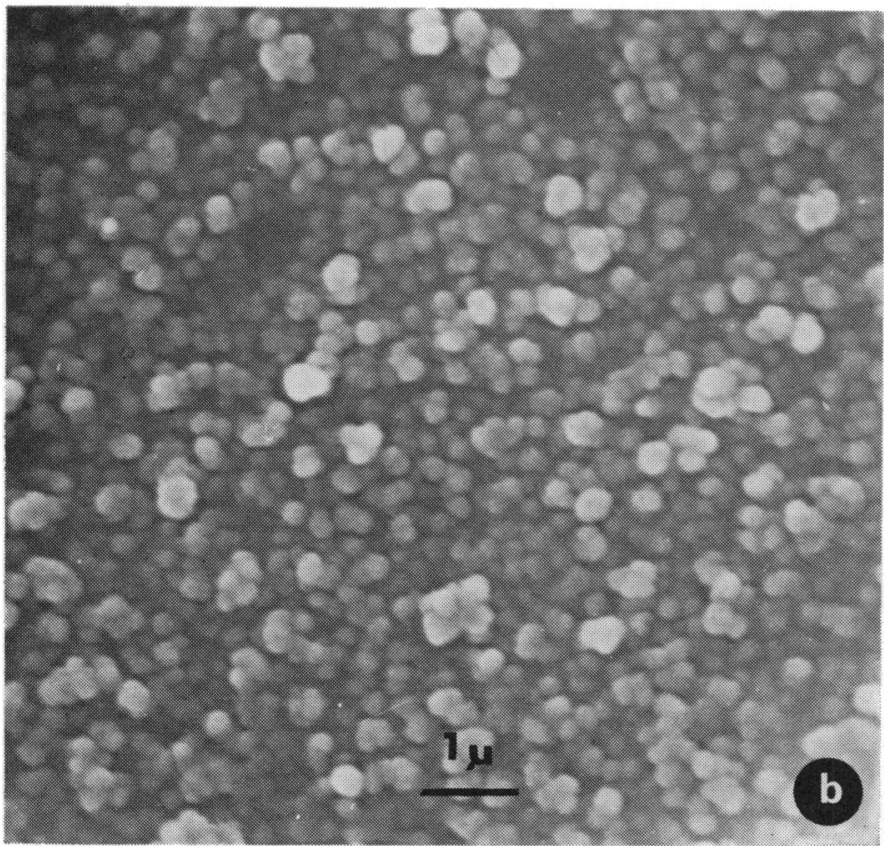

Fig. 6b. 


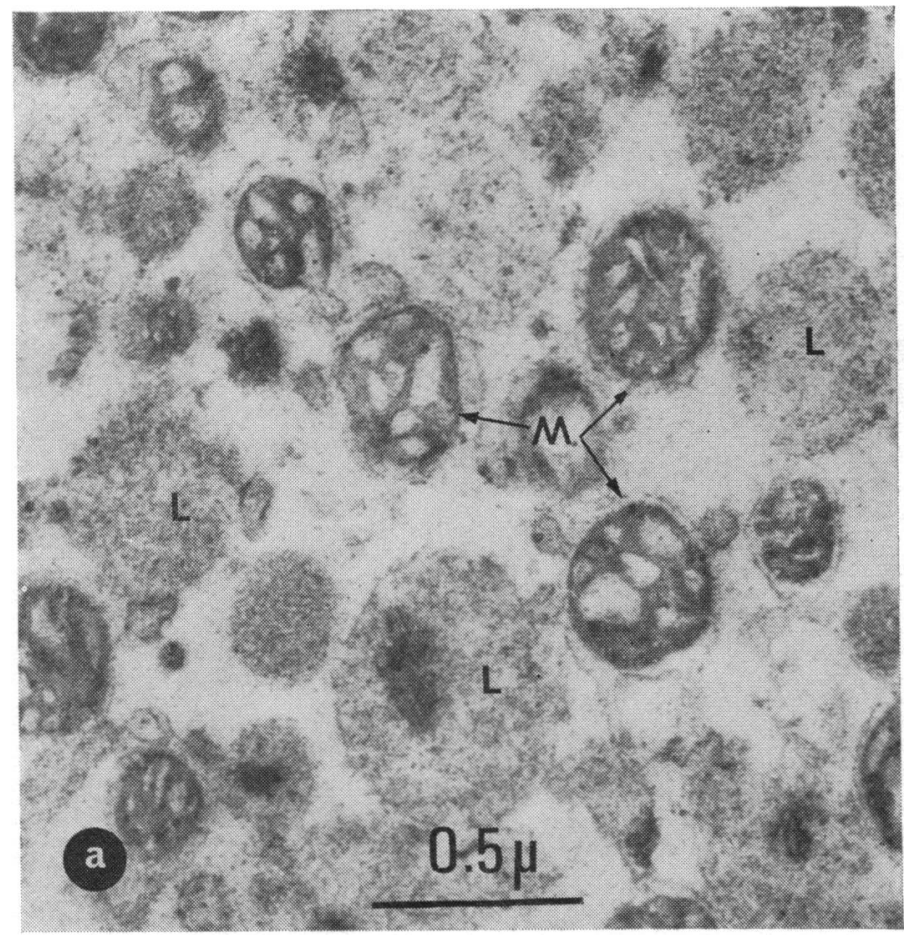

Fig. 7 Combined mitochondrial and lysosomal fraction.

a Transmission electron microscope and b Scanning electron microscope. The internal structure of each organelle is apparent, but their surfaces are smooth, and it is not possible to distinguish either particle in the scanning electron microscope.

Fig. 7a.

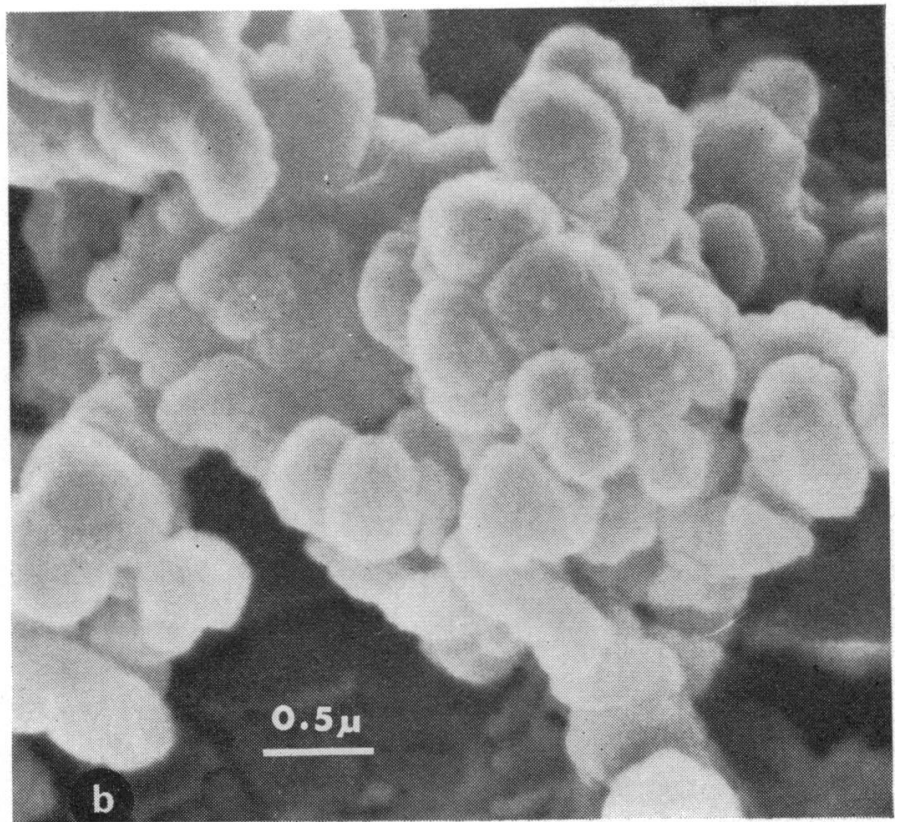

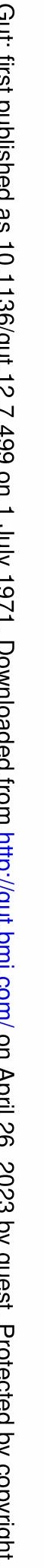




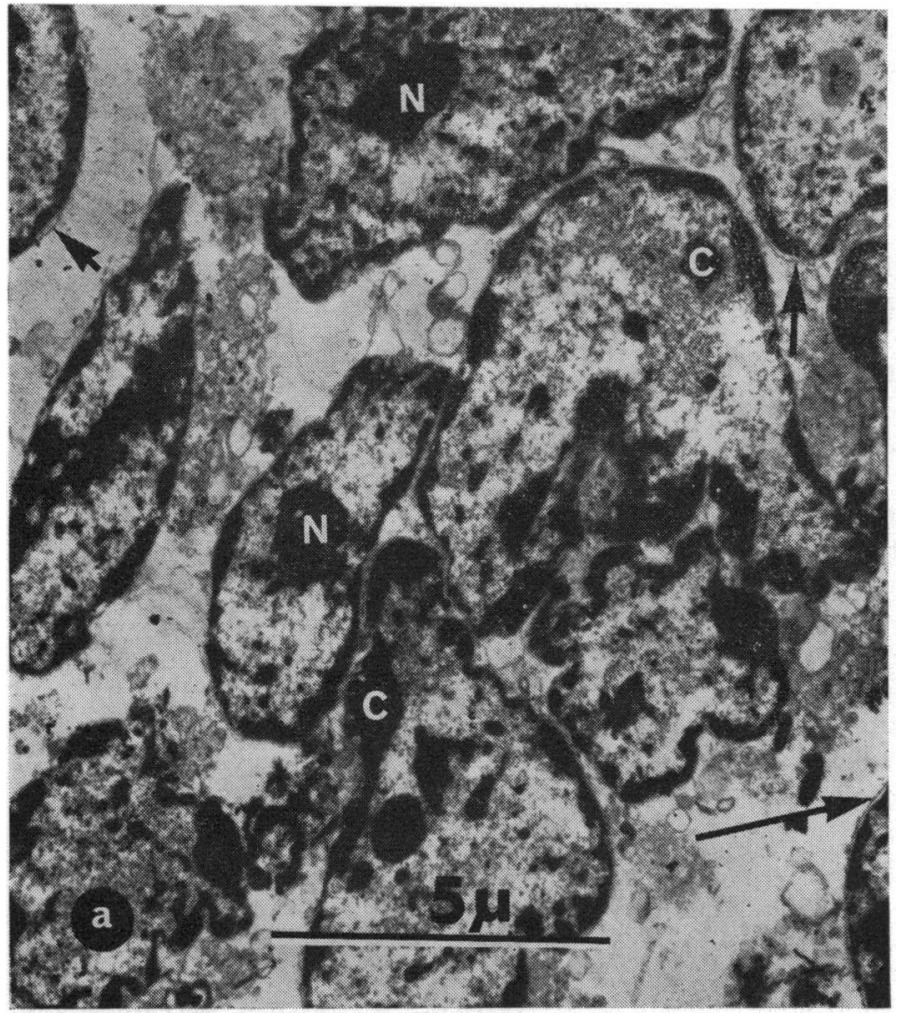

Fig. 8 Preparation of isolated nuclei. a Transmission electron microscope appearance showing fairly clean fraction. Nuclei have double membranes (arrows), nucleoli, $N$, and dense aggregations of chromatin material, $C$.

$b$ In the scanning electron microscope nuclei are of variable shape, but evidence of surface structure, eg, 'pores' is lacking.

Fig. 8 a.

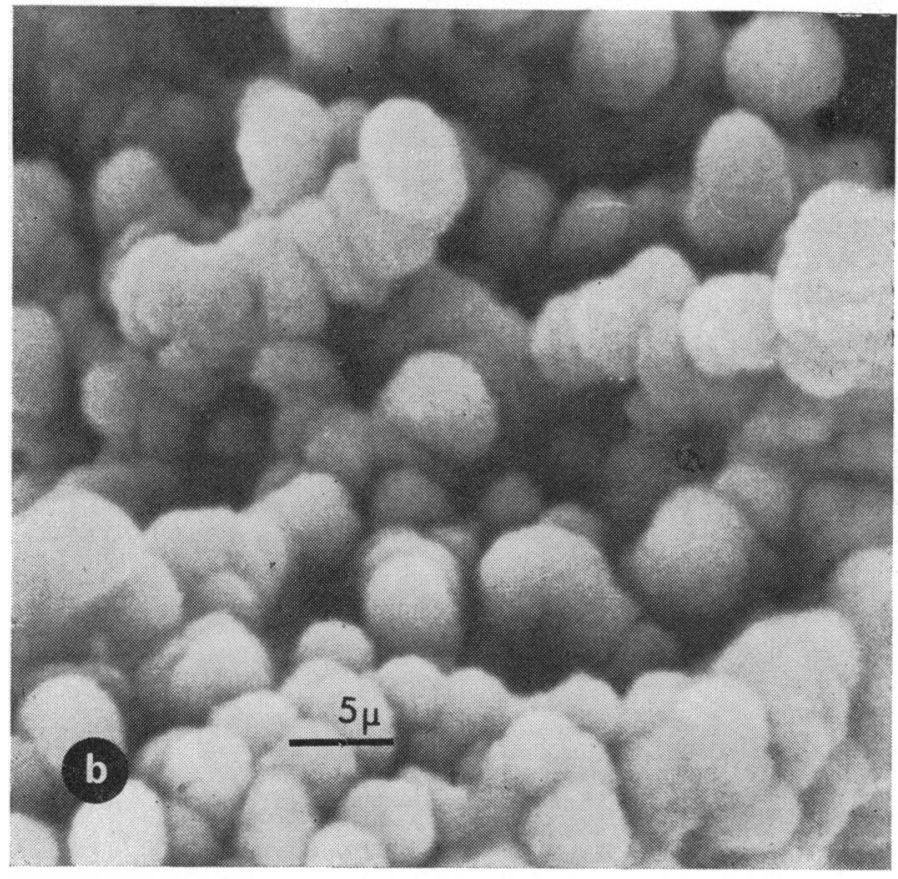

Fig. 8b. 
be seen, and the limiting membrane appeared as a smooth structure in the scanning electron microscope (Fig. 8b).

\section{Discussion}

These studies have provided some interesting observations on the structure of enterocytes, particularly in relation to their brush borders. Scanning electron microscopy is particularly useful for observing the external appearance of whole cells and for demonstrating the spatial arrangement of the microvilli.

Our observations indicate that the morphology of the cell is well preserved even after incubation for at least one hour. In particular, it is clear that the brush border retains its apical position and does not completely enfold the cells, as was suggested by Pink, Croft, and Creamer (1970). In comparing the appearance of the two brush border preparations, it is apparent that those prepared by the method of Eichholz and Crane (1965) are morphologically intact and are less contaminated by other subcellular organelles than those obtained by the technique of Hübscher et al (1965). These findings are in agreement with biochemical analyses of the same brush border preparations (Peters, 1970), which showed that the brush borders prepared by the Eichholz and Crane technique were much less contaminated with enzymes characteristic of other subcellular fractions than the brush borders obtained by the method of Hübscher et al (1965). In addition, the microvilli of the latter fractions showed a significant degree of fragmentation.

The appearances of microvilli of isolated enterocytes and of intact brush borders (each prepared by two methods) were studied with the scanning electron microscope. In these four preparations the microvilli were morphologically identical, and in addition, were similar to those seen in intact mucosa from human and rat jejunum (Swift and Marsh, 1968; Marsh and Swift, 1969). It is therefore apparent that the demonstration of microvilli is not due to artefacts in preparation, as suggested by Millington, Critchley, Tovell, and Pearson (1969).

One other advantage of the scanning electron microscope is that for the first time it has been possible to observe the undersurface of the intestinal epithelium. The cells are seen as finger-like projections which are separated from each other at their most basal aspects, suggesting that attachment to the subepithelial lamina (basement membrane) is necessary for the maintenance of the shape of the cell. In the transmission electron microscope the base of enterocytes is seen to be flat, while interdigitating processes commonly arise from their lateral cell walls, particularly in the zone just proximal to the lamina. Evidence for the occurrence of interdigitating processes was obtained in the scanning electron microscope and the study of other subcellular organelles did not provide any further information about their external morphology, and it seems that the technique of freeze-etching may be more useful for this purpose (Wrigglesworth, Packer, and Branton, 1970).

We thank Professor G. W. Causey (Royal College of Surgeons, London) for his kindness in allowing one of us (M.N.M.) the use of the transmission electron microscope, and Dr Alan Swift (Unilever Research Laboratory) for helpful discussions during the early part of this work.

We also would like to extend our gratitude to Professor C. C. Booth for his support and continued interest in this project. During this period T.J.P. was in receipt of an MRC clinical research fellowship.

\section{References}

Eichholz, A., and Crane, R. K. (1965). Studies on the organisation of the brush border in intestinal epithelial cells. I. This disruption of isolated hamster brush borders and density gradient separation of fractions. J. Cell Biol., 26, 687-691.

Evans, E. M., Wrigglesworth, J. M., Burdett, K., and Pover, W. F. R. Studies on epithelial cells from guinea pig small intestine 1. Isolation and morphology (personal communication).

Frasca, J. M., and Parks, V. R. (1965). A routine technique for double-staining ultra-thin sections using uranyl and lead salts. J. Cell Biol., 25, 157-161.

Haussler, M. R., Thompson, W. W., and Norman, A. W. (1969). The isolation of purified nuclear and chromatin fractions from chick intestinal mucosa. Exp. Cell Res., 58, 234-242.

Hübscher, G., West, G. R., and Brindley, D. N. (1965). Studies on the fractionation of mucosal homogenates from the small intestine. Biochem. J., 97, 629-642.

Marsh, M. N., and Swift, J. A. (1969). A study of the small intestinal mucosa using the scanning electron microscope. Gut, 10, 940-949.

Millington, P. F., Critchley, D. R., Tovell, P. W. A., and Pearson, R. (1969). Scanning electron microscopy of intestinal microvilli. J. Micr., 89, 339-344.

Párducz, B. (1967). Ciliary movement and co-ordination in ciliates. Int. Rev. Cytol., 21, 91-128.

Peters, T. J. (1970). The subcellular localisation of di- and tri-peptide hydrolase activity in guinea-pig small intestine. Biochem. J., 120, $195-203$.

Pink, I. J., Croft, D. N., and Creamer, B. (1970). Cell loss from small intestinal mucosa: a morphological study. Gut, 11, 217-222.

Sabatini, D. D., Bensch, K., and Barrnett, R. J. (1963). Cytochemistry and electron microscopy: the preservation of cellular ultrastructure and enzymatic activity by aldehyde fixation. J. Cell. Biol., 17, 19-58.

Small, E. B., and Marszalek, D. S. (1969). Scanning electron microscopy of fixed, frozen and dried Protozoa. Science, 163, 1064-1065.

Swift, J. A., and Marsh, M. N. (1968). Scanning electron microscopy of rat intestinal microvilli. Lancet, 2, 915-916.

Wrigglesworth, J. M., Packer, L., and Branton, D. (1970). Organisation of mitochondrial structure as revealed by freeze-etching. Biochim. biophys. Acta (Amst.), 205, 125-135. 